\title{
IMPULSE RESPONSE IDENTIFICATION BY ENERGY SPECTRUM METHOD USING GEOINFORMATION DATA IN CASE OF REMOTE SENSING IMAGES
}

\author{
A.Y. Denisova, V.V. Sergeyev \\ Samara National Research University, Samara, Russia
}

\begin{abstract}
In this paper a modification of spectral energy method of a linear observation model identification using data from geoinformation systems is described. Spectral energy method uses relation between energy spectra of original and output images. Original undistorted image is assumed to be unknown. The modification described in this paper supposes that boundaries of all image regions are known. It applies these boundaries to construct an image with energy spectrum similar to original undistorted one. The algorithm is considered in terms of remote sensing images, for which the boundaries of image regions can be presented as vector map data of the same territory and can be recieved from geoinformation systems.
\end{abstract}

Keywords: linear observation model, identification, geoinformation systems

Citation: Denisova AY, Sergeyev VV. Impulse response identification by energy spectrum method using geoinformation data in case of remote sensing images. CEUR Workshop Proceedings,2016; 1638: 290-295. DOI: 10.18287/1613-0073-2016-1638-290-295

\section{Introduction}

Linear observation model is often used to describe the process of image capturing in remote sensing systems. In many cases it is necessary to obtain an impulse response of distortion system using only observed image as input. This problem is called blind channel identification. There are several approaches to blind system identification, for example, solving convolution equation [1], using assumption about some statistical properties of the input image [2], estimating parametric models of impulse response [3]. The main disadvantages of these approaches are that they can not perform well with large images because of their computational complexity or instability in two dimensional case.

It is seemed to be a good decision for remote sensing data to use earlier developed in [4-6] energy spectrum method of impulse response identification. The method allows to estimate smoothing impulse response in two dimensional case by means of calcula- 
tion of observed image autocorrelation function and energy spectrum. For large data volumes, which are typical for remote sensing data, required characteristics are statistically stable and there are fast algorithms based on Fourier transform, which allow to compute them effectively.

\section{$1 \quad$ Energy spectrum method}

In accordance with the linear observation model the relation between original image and distorted image is described as follows:

$$
y\left(n_{1}, n_{2}\right)=\sum_{k_{1}, k_{2}=-K}^{K} h\left(k_{1}, k_{2}\right) x\left(n_{1}-k_{1}, n_{2}-k_{2}\right)+v\left(n_{1}, n_{2}\right), n_{1}, n_{2}=\overline{0, N-1},
$$

where $y\left(n_{1}, n_{2}\right)$ is observed image, $h\left(k_{1}, k_{2}\right)$ is unknown impulse response, $x\left(n_{1}, n_{2}\right)$ is unknown original image without distortions, $v\left(n_{1}, n_{2}\right)$ is statistically independent from signal white noise, $N$ is image size. The model includes an assumption that impulse response is normalized: $\sum_{k_{1}, k_{2}=-K}^{K} h\left(k_{1}, k_{2}\right)=1$.

Energy spectrum method is based on the relation between energy spectra of original and observed images [7]:

$$
\Phi_{Y}\left(e^{i \omega_{1}}, e^{i \omega_{2}}\right)=\mid H\left(e^{i \omega_{1}}, e^{i \omega_{2}}\right)^{2} \Phi_{X}\left(e^{i \omega_{1}}, e^{i \omega_{2}}\right)+D_{V}
$$

where $\Phi_{Y}\left(e^{i \omega_{1}}, e^{i \omega_{2}}\right)$ is energy spectrum of observed image, $\Phi_{X}\left(e^{i \omega_{1}}, e^{i \omega_{2}}\right)$ is energy spectrum of undistorted image, $H\left(e^{i \omega_{1}}, e^{i \omega_{2}}\right)$ is frequency response, $D_{V}$ is dispersion of white noise, and $\omega_{1}, \omega_{2}$ are cyclic frequencies.

In real remote sensing systems negative frequency response components corresponds to frequency values higher than maximum frequency presented in a periodic spectrum of sequence, because they are designed to take into account blur produced by detector and they have sampling step agreed with the blur size. Therefore, the following expression can be applied to estimate frequency response (2):

$$
H\left(e^{i \omega_{1}}, e^{i \omega_{2}}\right)=\sqrt{\frac{\Phi_{Y}\left(e^{i \omega_{1}}, e^{i \omega_{2}}\right)-D_{V}}{\Phi_{X}\left(e^{i \omega_{1}}, e^{i \omega_{2}}\right)} .}
$$

Impulse response can be obtained through inverse Fourier transform of frequency response. The method of impulse response identification using expression (3) is called as spectral energy method. The key issues of the method are how to estimate observed image energy spectrum, noise dispersion and energy spectrum of unknown undistorted image. To compute energy spectrum of observed image classical methods of digital spectral analysis can be used [15]. There also many methods for noise dispersion evaluation. In this study averaging of observed energy spectrum values correspond- 
ing to high frequencies was used, brief description of it can be found in [10]. The most interesting subproblem is estimation of unknown undistorted image energy spectrum.

\section{Energy spectrum method using GIS data}

The modification of energy spectrum method is a nonparametric method and it requires following assumptions to be legal:

1. The brightness of undistorted image for any image section is piecewise-constant function. This assumption seems natural for remote sensing images, since the objects on the Earth has clear boundaries.

2. The impulse response is smoothing and affects brightness only on the edges of regions with constant brightness levels.

It is known that the autocorrelation function properties are defined by the intensity of brightness leaps in each direction on the image. Estimation of undistorted autocorrelation function can be achieved from sharpened image with restored border information. Then such estimation can be used to compute undistorted energy spectrum.

The fact, that remote sensing images represent a part of Earth surface, makes possible to use electronic map data about boundaries of the objects on the image. Geoinformation systems are the most widespread and well organized electronic map source. The common way to store objects' boundaries is a vector map, which includes a set of spatial coordinates for each object. Vector map can be transformed into raster with given accuracy. Then raster is used as boundary mask to construct piecewise-constant image with sharp edges from the observed one. Received image is used to get energy spectrum similar to energy spectrum of unknown original image.

Further, it is assumed that noise dispersion is has been already estimated. Therefore, proposed modification can be written as follows:

1. Generate raster mask $D\left(m_{1}, m_{2}\right), 0 \leq m_{1}, m_{2} \leq M N-1$ of regions' borders by vector map. The raster mask should be in $M$ times larger than observed image to represent borders more precisely. For each region $D_{i}$ in mask the pixels are colored with the index $i=1, \ldots, I$ of region in vector map, where $I$ is the total number of image objects on image. It is obvious that raster mask should have the same reference system as the observed image.

2. Increase the size of observed image $y\left(n_{1}, n_{2}\right), 0 \leq n_{1}, n_{2} \leq N-1$ though the bilinear interpolation with step $1 / M: y\left(m_{1}, m_{2}\right), 0 \leq m_{1}, m_{2} \leq M N-1$.

3. Construct image with sharp edges $\hat{x}\left(m_{1}, m_{2}\right), 0 \leq m_{1}, m_{2} \leq M N-1$ by averaging of observed image pixels within the boundaries from the vector map:

$$
\hat{x}\left(m_{1}, m_{2}\right)=y_{i}, m_{1}, m_{2} \in D_{i}, i=\overline{1, I}, y_{i}=\frac{1}{\left|D_{i}\right|} \sum_{m_{1}, m_{2} \in D_{i}} y_{i n}\left(m_{1}, m_{2}\right),
$$

where $\left|D_{i}\right|$ is amount of pixels, corresponding to $i$-th mask region. 
4. Estimate energy spectrum of the original undistorted image $\Phi_{X}\left(e^{i \omega_{1}}, e^{i \omega_{2}}\right)$ as energy spectrum $\hat{\Phi}_{X}\left(e^{i \omega_{1}}, e^{i \omega_{2}}\right)$ of the image $\hat{x}\left(m_{1}, m_{2}\right)$ received on step 3 .

Compute energy spectrum of observed image. It should be calculated using observed image before interpolation. However, formula (3) supposes that both spectra have the same sampling step. To get energy spectra of observed image with smaller sampling step zeroes should be added in frequency domain. It corresponds to interpolation with sinc basis functions in spatial domain [7]. If the influence of other spectrum periods is low, an energy spectrum of sinc-interpolated signal with sampling step $T$ takes the form: $\Phi_{i n}\left(\Omega_{1}, \Omega_{2}\right)=\frac{1}{T^{2}} \Phi_{i n}\left(\Omega_{1}, \Omega_{2}\right)$, where $\Phi_{i n}\left(\Omega_{1}, \Omega_{2}\right)$ is interpolated energy spectrum and $\Omega_{1}, \Omega_{2}$ are frequencies. So the energy spectrum $\hat{\Phi}_{Y}\left(e^{i \omega_{1}}, e^{i \omega_{2}}\right)$ used in expression (3) is a result of multiplying observed image energy spectrum $\Phi_{Y}\left(e^{i \omega_{1}^{\prime}}, e^{i \omega_{2}^{\prime}}\right)$ on $M^{2}$ and adding zeroes to it up to size $M N$. The factor $M^{2}$ corresponds to sampling step $1 / M$.

5. Calculate frequency response using formula (3).

6. Estimate impulse response though the inverse Fourier transform of received frequency response.

Interpolation of observed image on step 4 is required to provide boundaries more precisely. Borders accuracy is highly correlated with the energy spectrum estimation quality. To compute energy spectra on the stages 4 and 5 any standard method of digital spectral analysis can be applied [8].

\section{Experimental Research}

The experimental research was made for set of mosaic images. Firstly, to model undistorted input image the images with fine sampling step were generated. Correlation coefficient of neighbor pixels for undistorted images equaled to 0.99 . Raster masks of boundaries were obtained simultaneously with the original mosaic images and had the size of $4096 \times 4096$ pixels. After the original images set had been prepared the set of distorted images was constructed according to the linear observation model described above. Distortions were made with the following impulse response corresponding to the impulse response of MODIS sensor built in Terra and Aqua spacecrafts [9]:

$h_{M}\left(m_{1}, m_{2}\right)=h_{1}\left(m_{1}, m_{2}\right) * * h_{2}\left(m_{1}, m_{2}\right) * * h_{3}\left(m_{1}, m_{2}\right)$,

where $\quad * * \quad$ is a convolution, $\quad h_{1}\left(k_{1}, k_{2}\right)=A \exp \left(-\left(k_{1}^{2}+k_{2}^{2}\right) / 2 \sigma^{2}\right)$, $h_{2}\left(k_{1}, k_{2}\right)=\operatorname{rect}\left(k_{1} / w\right) \operatorname{rect}\left(k_{2} / w\right), \quad h_{3}\left(k_{1}, k_{2}\right)=\operatorname{rect}\left(k_{1} / s\right),-K \leq k_{1}, k_{2} \leq K$. In our notation the parameters of the impulse response were $\sigma=w=s=8$.

Generated impulse response was used as etalon in comparison to the restored impulse responses. The sampling step of the impulse response was the same as for the fine images. 
Finally, observed image was obtained as a result of convolution of etalon impulse response and original image, sampling with bigger sampling step than sampling step of original image and putting of independent "white" noise into final image. Observed images size was $512 \times 512$ pixels. Reconstruction was made with bilinear interpolation on the stage 2 with the step 0,125 and with parameter $M=8$.

The example of observed image is presented in fig. 1 . The results of impulse response estimation in case of signal to noise ratio equal to 120 are shown in fig. 2 .

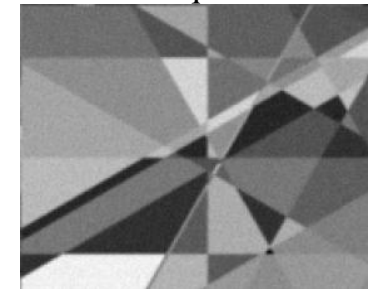

Fig. 1. The fragment of observed image

a)

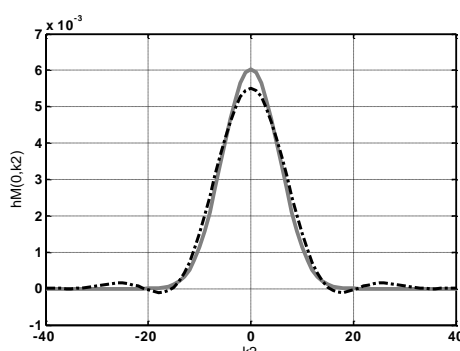

b)

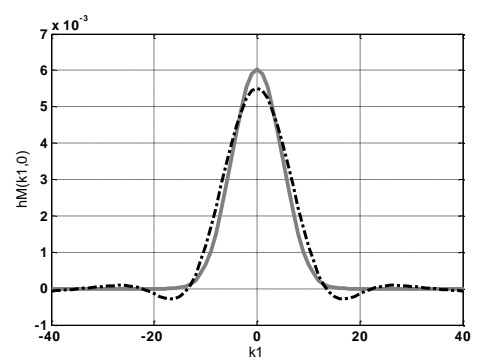

Fig. 2. The central section in a) transverse and b) longitudinal directions of ideal (gray) and restored (black) impulse responses

The error of impulse response restoration was estimated by normalized root mean square error:

$$
\varepsilon=\frac{1}{(2 K+1) h(0,0)} \sqrt{\sum_{k_{1}, k_{2}=-K}^{K}\left(h\left(k_{1}, k_{2}\right)-\hat{h}\left(k_{1}, k_{2}\right)\right)^{2}},
$$

where $h\left(k_{1}, k_{2}\right)$ is ideal impulse response, $\hat{h}\left(k_{1}, k_{2}\right)$ is estimated impulse response. It (5) is considered as relative error.

Table 1 contains average values $\varepsilon$ of the error and its standard deviation $\sigma_{\varepsilon}$ in cases with different values of signal to noise ratio $d$ in observed images.

Table 1. Average value and standard deviation

\begin{tabular}{cccccc}
\hline \multicolumn{2}{c}{$\mathbf{d = 2 5 0}$} & \multicolumn{2}{c}{$\mathbf{d = 1 2 0}$} & \multicolumn{2}{c}{$\mathbf{d = 1 5}$} \\
\hline$\varepsilon$ & $\sigma_{\varepsilon}$ & $\varepsilon$ & $\sigma_{\varepsilon}$ & $\varepsilon$ & $\sigma_{\varepsilon}$ \\
\hline $\mathbf{0 . 0 0 3 9}$ & $\mathbf{0 . 0 0 0 1}$ & $\mathbf{0 . 0 0 4 5}$ & $\mathbf{0 . 0 0 0 1}$ & $\mathbf{0 . 0 0 7 5}$ & $\mathbf{0 . 0 0 0 1}$ \\
\hline
\end{tabular}


From table 1 it can be seen that in the error is less than $1 \%$. So proposed method allows to reconstruct impulse response with high accuracy even in presence of noise with SNR value more than 15 .

\section{Conclusion}

Experiments in our study show that proposed method can be applied to impulse response restoration in remote sensing images. The main advantage of the proposed method is that it allows to reconstruct impulse an response with sampling step smaller than the sampling step of observed image.

\section{Acknowledgements}

The research was financially supported by RSF, grant №16-37-00043_mol_a «Development of methods of using data from geoinformation systems in remote sensing data processing», grant №16-29-09494 ofi_m «Methods of computer processing of multispectral remote sensing data for vegetation areas detection in special forensics».

\section{References}

1. Fursov VA. Image restoration using filters with finite impulse response by means of direct identification of inverse tract. Computer Optics, 1996: 16: 103-108. [in Russian]

2. Goriachkin OV, Erina EI. Blind channel identification by manifolds of given correlation generated by random polynoms. Achievements of Modern Radioelectronics, 2008; 8: 7077. [in Russian]

3. Bavrina AY, Myasnikov VV, Sergeyev AV. Parametrical identification of opticalelectronic tract of optical remote sensing system. Computer Optics, 2011; 35(4): 500-507. [in Russian]

4. Sergeyev VV, Denisova AY. Spectral-Energy Identification Method of the Linear Observation Model for Remote Sensing of the Earth. Pattern Recognition and Image Analysis, 2011; 21(2): 321-323.

5. Sergeyev VV, Denisova AY. Iterative method of piecewise-constant image restoration in case of regions border knowledge. Computer Optics, 2013; 37(2): 239-243. [in Russian]

6. Sergeyev VV, Denisova AY. Spectral energy identification method of the linear observation model in the absence of a covariance function model. Pattern Recognition and Image Analysis, 2014; 24(4): 561-565.

7. Soifer VA. Computer Image Processing, Part II: Methods and algorithms. VDM Verlag, 2009.

8. Marple JS, Lawrence. Digital spectral analysis with applications. Englewood Cliffs, NJ, Prentice-Hall, Inc., 1987; 512 p.

9. Schowengerdt RA. Remote sensing: models and methods for image processing. Academic press, 2006. 